\title{
ATTITUDES OF NEONATAL NURSES TO RESEARCH AND THEIR ROLE IN THE RESEARCH
} PROCESS

\author{
C. Soto ${ }^{1}$, H. Terry ${ }^{1}$, H. Pandya ${ }^{1}$, D.J. Field ${ }^{2}$, V. Kairamkonda ${ }^{3}$, E.M. Boyle ${ }^{2}$ \\ ${ }^{1}$ Infection, Immunity and Inflammation, ${ }^{2}$ Department of Health Sciences, University of Leicester, ${ }^{3}$ Neonatal \\ Unit, University Hospitals Leicester, Leicester, UK
}

Background: Little is known about how nursing staff view their participation in clinical research, especially within neonatal care. It has been suggested that nurses are generally supportive of research, although participation may be influenced by time pressures, care of their patient, and limited experience of research. We designed a qualitative study to explore these influences.

Objective: To explore neonatal nurses views of clinical research, and their perception of nurses' role in the research process.

Method: Thirty-one nurses from a neonatal service participated in semi-structured interviews, which were analysed through the constant comparative method, assisted by NVivo software.

Results: Nurses are supportive of clinical research, and recognise its importance in delivering the best care to their patients. Despite appreciating the benefits of research involvement, nurses see their primary role as advocates for the babies and their families, promoting clinical care above research. Nurses do not view themselves as involved in research, although they perform supportive tasks (such as collecting samples, recording data), and see this as part of their caring role. In particular, research is seen as an integral part of working in an intensive care unit, although nurses experience challenges in accessing and assimilating research information.

Conclusions: Nurses play an important research role by promoting studies, collecting data, and acting as advocates for their patients. However, nurses do not feel part of the research process, which impacts on their participation. Further research is needed to enhance nurse engagement, and thus improve the success of clinical research projects. 